\title{
ATIVIDADE FÍSICA DE IDOSOS E A PROMOÇÃO DA SAÚDE NAS UNIDADES BÁSICAS
}

\author{
PHYSICAL ACTIVITY OF ELDERLY PEOPLE AND PROMOTION OF HEALTH AT BASIC UNITS
}

ACTIVIDAD FÍSICA DE ADULTOS MAYORES Y LA PROMOCIÓN DE LA SALUD EN LAS UNIDADES BÁSICAS

\author{
Paulo Henrique Vilela Oliveira de Sá ${ }^{1}$ \\ Geraldo Cunha Cury ${ }^{2}$ \\ Liliane da Consolação Campos Ribeiro ${ }^{3}$
}

Resumo $\mathrm{O}$ estudo teve como objetivo identificar e descrever as ações de promoção à saúde relacionadas à atividade física de idosos nas unidades básicas de saúde, assim como a percepção dos responsáveis sobre essa prática. Tratou-se de uma pesquisa transversal, de abordagem quantitativa, realizada em Diamantina, Minas Gerais, em 2012. Um instrumento com 28 assertivas, organizado na forma de escala Likert, foi utilizado durante as entrevistas com 28 responsáveis por tais ações. Constataram-se 11 ações educativas de promoção da saúde relacionadas à atividade física para idosos, cinco de responsabilidade dos professores e alunos de uma instituição pública de ensino e seis de responsabilidade dos profissionais das equipes de saúde da família. As ações observadas foram 'grupo de caminhada e exercícios (alongamentos)', 'grupo de coluna' e 'Qigong'. Os participantes demonstraram seu entendimento sobre os benefícios das atividades físicas para os idosos, o impacto dessas ações na saúde pública e a necessidade de permanente capacitação.

Palavras-chave envelhecimento; educação em saúde; educação continuada; atividade física; saúde da família.
Abstract The study aimed to identify and describe health promotion actions related to physical activity among elderly people at basic health units and the perception of those responsible for this practice. This was a cross-sectional survey using a quantitative approach carried out in Diamantina, Minas Gerais State, Brazil, in 20112. A 28-questions survey, organized in the form of a Likert scale, was used during the interviews carried out among 28 people in charge of such actions. Eleven educational health promotion actions related to physical activity among elderly people were found, five of which under the responsibility of teachers and students of a public school, while six under headed by professionals from the family health teams. The actions observed were 'walk and exercise group (stretching), ' 'back group,' and 'Qigong.' Participants demonstrated their understanding of the benefits afforded by phy-sical activity for the elderly, the impact these actions have on public health, and the need for ongoing training.

Keywords aging; health education; continuing education; physical activity; family health. 


\section{Introdução}

O envelhecimento humano é um fenômeno natural, social, irreversível e mundial, que não pode ser caracterizado apenas pela degeneração biológica, mas como resultado dessa situação relacionada com problemas e limitações de ordem econômica, de condições políticas, históricas e socioculturais que singularizam esse processo (Santos, Foroni e Chaves, 2009; Melo et al., 2009).

O período entre 1970 e 2025 é considerado a era do envelhecimento. No Brasil, é considerado idoso todo indivíduo que possui 60 anos ou mais de idade (Brasil, 2003; Góis e Veras, 2010). Estima-se que até 2060 o número de idosos no Brasil chegue a 34\%; no país, atualmente, $13 \%$ da população tem acima de 60 anos (Instituto Brasileiro de Geografia e Estatística, 2014).

Com o aumento proporcional do número de idosos, existe a necessidade da adequação e preparo dos serviços básicos de saúde, visando a sua estruturação, formação e qualificação profissional para o atendimento dessa nova demanda (Siqueira et al., 2009), uma vez que a população idosa é grande usuária de serviços de saúde (Salin et al., 2011; Pilger, Menon e Mathias, 2013).

Um potencial serviço de saúde que atende esses idosos é a Estratégia Saúde da Família (ESF), implementada como uma proposta de reorganização do Sistema Único de Saúde (SUS) no âmbito da atenção primária à saúde (APS) (Conill, 2008). O foco de atuação da ESF está voltado para ações principalmente de cunho educativo, de promoção da saúde, junto aos indivíduos e às coletividades em sua totalidade, sem distinção de crenças, cultura, grau de escolaridade ou situação econômica e social. Busca sempre despertar nas pessoas o interesse pelo conhecimento das diversas formas de obter adequadas condições de saúde e de exercer a plena cidadania (Roecker e Marcon, 2011).

Nesse contexto, programas de promoção da saúde relacionados à atividade física se apresentam como uma estratégia na busca de um envelhecimento saudável e devem ser priorizados nas intervenções realizadas nas unidades de saúde da família, uma vez que, além dos benefícios físicos, psicológicos e sociais, podem gerar grande economia no setor de saúde (Siqueira et al., 2009; Rocha et al., 2013; Gonzaga et al., 2011).

Apesar da importância do assunto, ainda são escassos os estudos sobre avaliação da prática de atividade física para idosos como ação de promoção da saúde no entendimento dos profissionais das ESF (Salin et al., 2011). O conhecimento dessa temática poderá subsidiar a implementação de políticas públicas e atividades de ensino voltadas para os profissionais que trabalham nessas equipes.

Com base no exposto, o objetivo desta investigação foi identificar e descrever as ações de promoção à saúde relacionadas à atividade física de idosos nas unidades básicas de saúde (UBSs) do município de Diamantina, no estado de Minas Gerais, e a percepção de seus responsáveis sobre essa prática. 


\section{Metodologia do estudo}

Esta pesquisa 4 foi realizada na cidade de Diamantina, estado de Minas Gerais, localizada a 292 quilômetros da capital, Belo Horizonte. Em 2012, o município contava com oito equipes de saúde da família, com cobertura em torno de $75 \%$ da população, dois hospitais e uma policlínica. O cenário do estudo foram as suas oito equipes de saúde da família.

Tratou-se de um estudo quantitativo descritivo transversal, no qual as informações foram coletadas por meio de um instrumento desenvolvido pelos próprios pesquisadores, baseado em aspectos metodológicos de escala atitudinal ou Likert e referencial teórico sobre o assunto pesquisado nas bases de dados Literatura Latino-Americana e do Caribe (Lilacs) e Scientific Electronic Library Online (SciELO), por meio dos descritores envelhecimento, atividade física, saúde da família.

O instrumento de análise foi constituído por 28 assertivas divididas em quatro dimensões: benefícios da atividade física para os idosos; incorporação do conceito de atividade física na prática profissional como ação de promoção da saúde para idosos; impacto das ações de promoção da saúde em atividade física para idosos na saúde pública; e necessidade de capacitação/treinamento para desenvolvimento de ações em promoção da saúde relacionadas à atividade física para indivíduos idosos. Cada dimensão apresentava sete assertivas com quatro opções de resposta: concordo totalmente; concordo parcialmente; discordo totalmente; e discordo parcialmente.

Participaram do estudo os responsáveis nas equipes de saúde da família pelas ações de promoção à saúde relacionadas à prática de atividade física para idosos, indicados pelo coordenador da equipe, durante uma visita do pesquisador nas próprias unidades de saúde, no período de junho a setembro de 2012, em horário predeterminado com os entrevistados.

As informações coletadas com relação ao objetivo proposto por este estudo foram digitadas em um banco de dados no software Epi Info versão 6.04 for Windows, a partir do qual realizou-se a análise de frequência das variáveis categóricas e descritivas das variáveis quantitativas.

Durante toda a pesquisa, respeitaram-se os aspectos éticos estabelecidos pelo Conselho Nacional de Saúde. A fim de também respeitar a privacidade dos profissionais, as unidades de saúde foram identificadas por números, e os profissionais, pela categoria profissional. A pesquisa foi aprovada pelo Comitê de Ética em Pesquisa da Universidade Federal dos Vales do Jequitinhonha e Mucuri com o número 015/2012. Todos os participantes receberam e assinaram o termo de consentimento livre e esclarecido. 


\section{Atividade física desenvolvida por idosos na atenção básica}

A área urbana do município de Diamantina possuía oito equipes de saúde da família em 2012, cada equipe em uma UBS. Duas equipes não ofereciam aos seus usuários nenhuma ação de promoção da saúde relacionada à atividade física para idosos.

As respectivas UBSs, as atividades e os responsáveis que desenvolviam ações relacionadas à prática da atividade física encontram-se no Quadro 1.

Quadro 1

\begin{tabular}{|c|c|c|}
\hline UBS & Ações desenvolvidas nas equipes de saúde da família & Responsáveis \\
\hline 1 & $\begin{array}{l}\text { Qigong (atividade física baseada em técnicas corporais, marciais e terapêuticas chinesas) } \\
\text { Grupo de caminhada e exercícios (alongamentos) } \\
\text { Grupo de caminhada e exercícios (alongamentos) }\end{array}$ & $\begin{array}{l}1 \text { professor do curso de educação física } \\
1 \text { enfermeira } \\
9 \text { alunos do curso de fisioterapia }\end{array}$ \\
\hline 2 & Não apresentou nenhuma ação de promoção da saúde relacionada com atividade física & \\
\hline 3 & Não apresentou nenhuma ação de promoção da saúde relacionada com atividade física & \\
\hline 4 & Grupo de caminhada e exercícios (alongamentos) & 1 enfermeira e 1 agente comunitário de saúde \\
\hline 5 & Grupo de caminhada e exercícios (alongamentos) & $\begin{array}{l}1 \text { enfermeira, } 2 \text { técnicos de enfermagem e } \\
5 \text { agentes comunitárias de saúde }\end{array}$ \\
\hline 5 & Grupo de caminhada e exercícios (alongamentos) & $\begin{array}{l}9 \text { alunos do curso de fisioterapia e } \\
1 \text { professor do curso de fisioterapia }\end{array}$ \\
\hline 6 & Grupo de coluna (alongamentos e exercícios específicos para a coluna vertebral) & 1 enfermeira e 1 fisioterapeuta do NASF \\
\hline 6 & Grupo de caminhada e exercícios (alongamentos) & 9 alunos do curso de fisioterapia \\
\hline 7 & Grupo de caminhada e exercícios (alongamentos) & 9 alunos do curso de fisioterapia \\
\hline 7 & Grupo de caminhada e exercícios (alongamentos) & Fisioterapeuta do NASF \\
\hline
\end{tabular}

Fonte: Pesquisa elaborada pelos autores.

Nota: NASF - Núcleo de Apoio à Saúde da Família; UBS - Unidade Básica de Saúde.

Os profissionais do serviço de saúde das equipes de saúde da família foram responsáveis por seis ações de promoção da saúde relacionadas à atividade física; os professores e estudantes dos cursos de graduação da área da saúde de uma instituição pública de ensino foram responsáveis por cinco atividades. Importante destacar a participação de uma instituição de ensino superior na prática das atividades, porém nenhuma das ações era realizada em parceria entre os profissionais de saúde das UBSs e os professores e alunos dos cursos de graduação; tratava-se de ações isoladas e marcadas por pouca comunicação entre elas, às vezes ocorrendo simultaneamente. 
Para os idosos, quase não havia opção de escolha de atividade física para participar, uma vez que apenas três tipos de ações foram relatados pelos entrevistados: 'Grupo de caminhada e exercícios/alongamentos', 'Grupo de coluna' (alongamentos e exercícios específicos para a coluna vertebral) e 'Qigong' (técnicas corporais, marciais e terapêuticas chinesas).

Em relação aos profissionais do serviço de saúde, professores e estudantes, foram encontrados 28 responsáveis por ações de promoção da saúde relacionadas à atividade física. Destes, nove $(32,1 \%)$ eram agentes comunitários de saúde (ACSs), nove $(32,1 \%)$ acadêmicos do curso de fisioterapia, cinco $(17,9 \%)$ enfermeiros, dois $(7,1 \%)$ técnicos de enfermagem, um $(3,6 \%)$ fisioterapeuta do NASF, um $(3,6 \%)$ professor do curso de fisioterapia e um $(3,6 \%)$ professor do curso de educação física.

A Tabela 1 apresenta o entendimento dos 28 responsáveis pela prática da atividade física em relação aos benefícios da atividade física para os idosos; a incorporação do conceito de atividade física na prática profissional como ação de promoção da saúde para idoso; e o impacto das ações de promoção da saúde em atividade física para idosos na saúde pública.

Tabela 1

Entendimento dos responsáveis pelas ações de promoção da saúde sobre as atividades físicas com idosos nas equipes de saúde da família, Diamantina, MG, 2012

\begin{tabular}{|c|c|c|c|c|}
\hline $\begin{array}{l}\text { Assertivas sobre a prática de } \\
\text { atividades físicas para idosos }\end{array}$ & $\begin{array}{l}\text { Concordo totalmente } \\
\qquad \mathrm{n}(\%)\end{array}$ & $\begin{array}{l}\text { Concordo parcialmente } \\
\qquad \mathrm{n}(\%)\end{array}$ & $\begin{array}{l}\text { Discordo parcialmente } \\
\qquad \mathrm{n}(\%)\end{array}$ & $\begin{array}{c}\text { Discordo totalmente } \\
\mathrm{n}(\%)\end{array}$ \\
\hline \multicolumn{5}{|l|}{ Benefícios } \\
\hline $\begin{array}{l}\text { 1. Promove benefícios } \\
\text { fisiológicos }\end{array}$ & $23(82,1)$ & $5(17,9)$ & - & - \\
\hline 2. Promove benefícios sociais & $24(85,7)$ & $2(7,1)$ & $2(7,1)$ & - \\
\hline $\begin{array}{l}\text { 3. Praticantes têm menor } \\
\text { chance de problemas } \\
\text { psicológicos }\end{array}$ & $24(85,7)$ & $3(10,7)$ & $1(3,6)$ & - \\
\hline $\begin{array}{l}\text { 4. Promove maior } \\
\text { independência funcional }\end{array}$ & $24(85,7)$ & $4(14,3)$ & - & - \\
\hline $\begin{array}{l}\text { 5. Auxilia na prevenção } \\
\text { de doenças }\end{array}$ & $23(82,1)$ & $5(17,9)$ & - & - \\
\hline $\begin{array}{l}\text { 6. Doentes crônicos } \\
\text { podem realizar }\end{array}$ & $11(39,5)$ & $17(60,5)$ & - & - \\
\hline $\begin{array}{l}\text { 7. Melhora a qualidade } \\
\text { de vida }\end{array}$ & $26(92,9)$ & $2(7,1)$ & - & - \\
\hline
\end{tabular}




\section{Continuação - Tabela 1}

Entendimento dos responsáveis pelas ações de promoção da saúde sobre as atividades físicas com idosos nas equipes de saúde

da família, Diamantina, MG, 2012

\begin{tabular}{|c|c|c|c|c|}
\hline $\begin{array}{l}\text { Assertivas sobre a prática de } \\
\text { atividades físicas para idosos }\end{array}$ & $\begin{array}{l}\text { Concordo totalmente } \\
\qquad \mathrm{n}(\%)\end{array}$ & $\begin{array}{l}\text { Concordo parcialmente } \\
\qquad \mathrm{n}(\%)\end{array}$ & $\begin{array}{l}\text { Discordo parcialmente } \\
\qquad \mathrm{n}(\%)\end{array}$ & $\begin{array}{l}\text { Discordo totalmente } \\
\text { n (\%) }\end{array}$ \\
\hline \multicolumn{5}{|l|}{$\begin{array}{l}\text { Incorporação na } \\
\text { prática profissional }\end{array}$} \\
\hline 8. Deve ser indicada & $23(82,1)$ & $3(10,7)$ & $1(3,6)$ & $1(3,6)$ \\
\hline $\begin{array}{l}\text { 9. É uma ação eficaz de } \\
\text { promoção da saúde }\end{array}$ & $24(85,7)$ & $3(10,7)$ & - & $1(3,6)$ \\
\hline $\begin{array}{l}\text { 10. Pode ser considerada } \\
\text { uma estratégia de educação } \\
\text { em saúde }\end{array}$ & $2(78,6)$ & $2(7,1)$ & $2(7,1)$ & $22(78,6)$ \\
\hline $\begin{array}{l}\text { 11. É de responsabilidade } \\
\text { exclusiva dos profissionais de } \\
\text { educação física ou } \\
\text { fisioterapeutas }\end{array}$ & $5(17,8)$ & $11(39,2)$ & $2(7,1)$ & $10(35,7)$ \\
\hline $\begin{array}{l}\text { 12. Não devem ser realizadas } \\
\text { em grupos }\end{array}$ & - & - & $6(21,4)$ & $22(78,6)$ \\
\hline $\begin{array}{l}\text { 13. Capacitam o idoso em } \\
\text { cuidar da própria saúde }\end{array}$ & $18(64,3)$ & $7(25,0)$ & $2(7,1)$ & $1(3,6)$ \\
\hline $\begin{array}{l}\text { 14. A comunidade não deve } \\
\text { se responsabilizar pela } \\
\text { promoção de saúde em } \\
\text { atividade física, pois é } \\
\text { obrigação do governo }\end{array}$ & - & - & $6(21,4)$ & $22(78,6)$ \\
\hline $\begin{array}{l}\text { Impacto das ações de } \\
\text { promoção à saúde }\end{array}$ & & & & \\
\hline $\begin{array}{l}\text { 15. Praticantes utilizam } \\
\text { menos medicamentos }\end{array}$ & $13(46,4)$ & $14(50,0)$ & $1(3,6)$ & - \\
\hline $\begin{array}{l}\text { 16. Não praticantes se } \\
\text { internam mais }\end{array}$ & $11(39,3)$ & $15(53,6)$ & $2(7,1)$ & - \\
\hline 17. Reduz custos com a saúde & $19(67,9)$ & $7(25,0)$ & $1(3,6)$ & $1(3,6)$ \\
\hline $\begin{array}{l}\text { 18. Diminui a prevalência } \\
\text { das morbidades }\end{array}$ & $2(7,1)$ & $2(7,1)$ & $5(17,9)$ & $19(67,9)$ \\
\hline $\begin{array}{l}\text { 19. Aumenta os custos } \\
\text { em saúde }\end{array}$ & $1(3,6)$ & $1(3,6)$ & $4(14,3)$ & $22(78,6)$ \\
\hline $\begin{array}{l}\text { 20. Não praticantes utilizam } \\
\text { mais serviços médicos }\end{array}$ & $17(60,7)$ & $9(32,1)$ & $2(7,1)$ & - \\
\hline $\begin{array}{l}\text { 21. Praticantes apresentam } \\
\text { menos doenças crônicas }\end{array}$ & $14(50,0)$ & $14(50,0)$ & - & - \\
\hline
\end{tabular}

Fonte: Pesquisa elaborada pelos autores. 
Percebe-se que houve concordância entre os entrevistados, com maior tendência à avaliação positiva (concordo totalmente e concordo parcialmente) no item de benefícios da prática de atividade física para idosos e impacto das ações na saúde pública. Por sua vez, a assertiva do item 11 ('São de responsabilidade exclusiva dos profissionais de educação física e/ou fisioterapeutas') foi a que obteve menores índices de concordância.

A Tabela 2 apresenta o entendimento dos entrevistados com relação à necessidade de capacitação para o desenvolvimento de ações de promoção da saúde relacionadas à atividade física para idosos. Ainda nesta tabela, foram abordadas questões como insegurança profissional, educação permanente, formas de trabalho com idosos, formas de trabalho em equipe, fragmentação do trabalho e construção coletiva de conhecimento.

Tabela 2

Entendimento dos responsáveis pela prática de atividade física sobre a capacitação profissional, Diamantina, MG, 2012

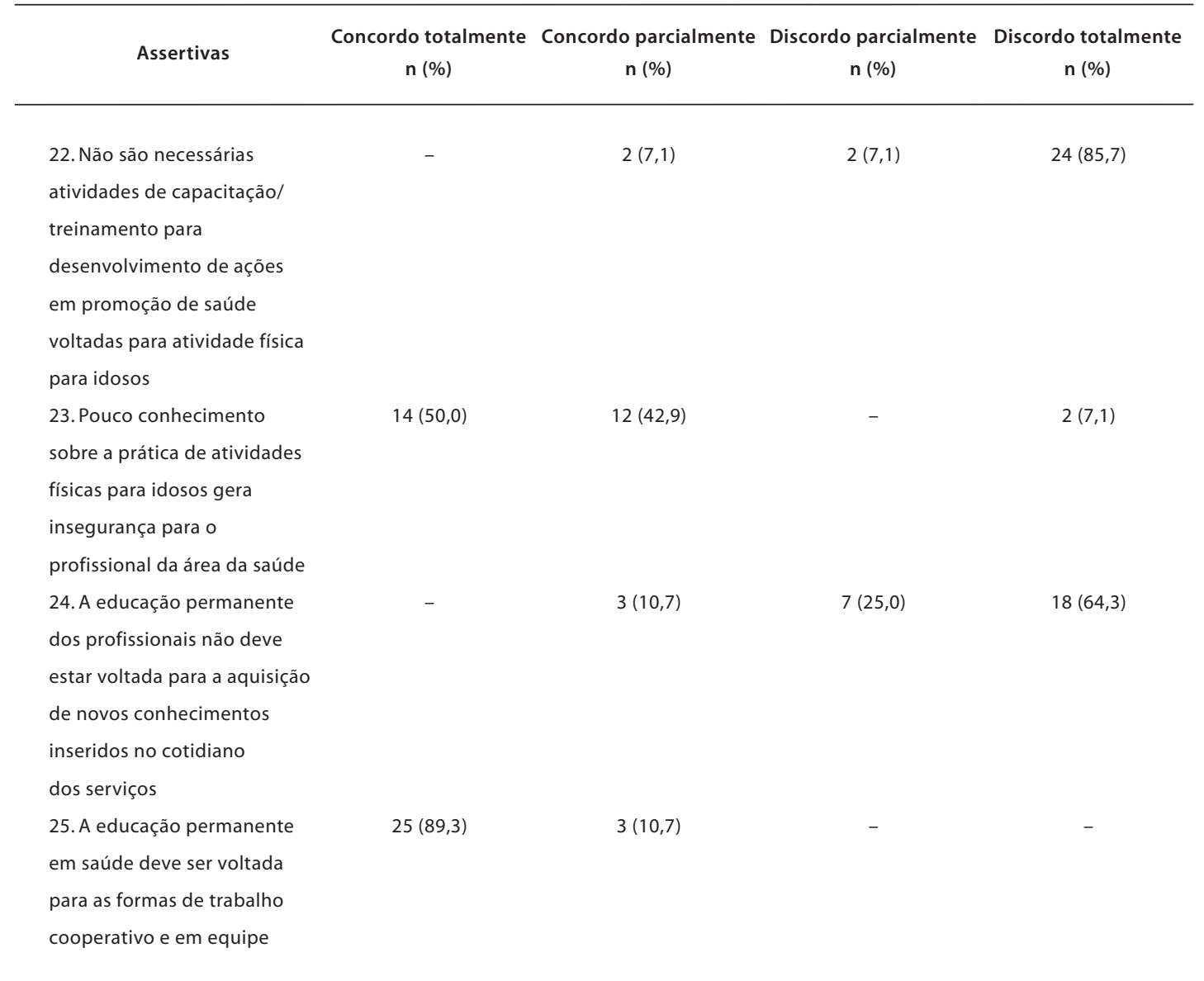




\begin{tabular}{|c|c|c|c|c|}
\hline Assertivas & $\begin{array}{l}\text { Concordo totalmente } \\
\text { n (\%) }\end{array}$ & $\begin{array}{l}\text { Concordo parcialmente } \\
\qquad \mathrm{n}(\%)\end{array}$ & $\begin{array}{c}\text { Discordo parcialmente } \\
\mathrm{n}(\%)\end{array}$ & $\begin{array}{l}\text { Discordo totalmente } \\
\text { n (\%) }\end{array}$ \\
\hline $\begin{array}{l}\text { 26. Um processo de } \\
\text { capacitação não deve se } \\
\text { contrapor à fragmentação, } \\
\text { divisão de trabalho e } \\
\text { normatização das tarefas }\end{array}$ & $10(35,7)$ & $8(28,6)$ & $5(17,9)$ & $5(17,9)$ \\
\hline $\begin{array}{l}\text { 27. A capacitação profissional } \\
\text { é mais efetiva quando } \\
\text { o foco é o profissional e } \\
\text { não a equipe }\end{array}$ & - & $7(25,0)$ & $5(17,9)$ & $16(57,1)$ \\
\hline $\begin{array}{l}\text { 28. A educação permanente } \\
\text { em saúde favorece } \\
\text { a construção coletiva } \\
\text { de conhecimento }\end{array}$ & $25(89,3)$ & $2(7,1)$ & - & $1(3,6)$ \\
\hline
\end{tabular}

Fonte: Pesquisa elaborada pelos autores.

\section{Resultados e discussão}

A ESF apresenta-se como importante cenário para a execução de múltiplas ações de saúde que procuram ir além do modelo curativo, centrado na doença. Ações de educação em saúde, prevenção e promoção da saúde com foco nas famílias e nas comunidades - e que adotam uma abordagem de análise da realidade local e de levantamento dos problemas biopsicossociais são cada vez mais requeridas na prática desses serviços (Tesser et al., 2011; Roecker e Marcon, 2011).

Na busca por ações de promoção da saúde que promovam autonomia e qualidade de vida para a população idosa, a atividade física regular aparece como uma ação fundamental (Salin et al., 2011; Rocha et al., 2013).

Este estudo indicou que os profissionais de saúde da rede de atenção, professores e estudantes dos cursos de graduação da área da saúde conseguem perceber os benefícios que um programa de atividade física regular pode trazer para a população idosa. Benefícios fisiológicos, sociais e psicológicos, prevenção de doenças, melhora na qualidade de vida e maior independência funcional foram amplamente citados e entendidos como importantes para os idosos. 
Pôde-se perceber que os entrevistados compreendiam que a atividade física é uma importante ação que deve ser desenvolvida com a população idosa, pois trata-se de uma eficaz ação de promoção e de educação em saúde, que pode capacitar os idosos a cuidarem da sua própria saúde. Entendiam também a importância da participação da comunidade nas ações de promoção da saúde. Entretanto, divergiam em relação à responsabilidade quanto ao desenvolvimento das ações de promoção da saúde relacionadas à prática de atividade física.

Segundo Lima e colaboradores (2010), a formação da maioria dos profissionais atuantes nos serviços de saúde ocorreu ou ocorre baseada na visão centrada na atenção às doenças, de forma fragmentada, pragmática e excessivamente biomédica. Tal formação dificulta o exercício da integralidade, o trabalho interdisciplinar e o desenvolvimento de ações que sejam propostas, planejadas e executadas com a participação de profissionais de diferentes áreas da saúde e das comunidades, como proposto pelo SUS.

Esse tipo de formação no campo da saúde raramente privilegia um enfoque que possibilite a estudantes e profissionais dos serviços o diálogo e a oportunidade de participação, ativa e conjuntamente, na construção de ações de promoção da saúde. Dessa forma, eles não reconhecem seu papel como membros ativos no processo de criação e implementação de tais ações. Ao delegar a responsabilidade da atividade a outros profissionais, perpetuam o modelo de divisão de tarefas e fragmentação do processo de trabalho.

Com relação ao impacto das ações de promoção relacionadas à atividade física para a idosos, percebeu-se que os profissionais dos serviços das ESFs, bem como os estudantes e professores dos cursos de graduação, entendiam a importância que tais ações causavam nos custos em saúde.

O aumento da proporção de idosos leva a um maior e mais frequente uso dos serviços de saúde; consequentemente, os gastos no setor poderão aumentar de forma substancial no Brasil, tendendo a emergir como um dos maiores desafios de gestão nos próximos anos (Pilger, Menon e Mathias, 2013).

A prevenção não é um custo e sim um investimento, desde que bem realizada. Portanto, é essencial ampliar as ações de promoção, prevenção e educação em saúde e criar estratégias para o retardamento de doenças e fragilidades, bem como implantar medidas que tenham como meta a manutenção da independência funcional e da autonomia do indivíduo idoso (Veras, 2012).

Implantar ações de promoção da saúde relacionadas à atividade física na atenção primária pode ser uma interessante alternativa de gestão em saúde, pois elas apresentam uma aceitável relação custo-benefício (Ramos, 2003). Proporcionar melhor qualidade de vida, funcionalidade e autonomia para esse contingente de idosos pode indicar uma interessante iniciativa para otimização dos recursos e controle dos gastos em saúde. Assim, é importante destacar que duas UBSs não desenvolviam nenhuma atividade e as outras seis se resumiam a caminhadas, Qigong e grupo de coluna. 
A ESF requer dos profissionais competências e habilidades muitas vezes esquecidas no processo de formação profissional (Cardoso, Murad e Bof, 2005). Os entrevistados expressaram de maneira clara e contundente seus anseios quanto à necessidade de capacitação para desenvolveram ações de promoção da saúde relacionadas à atividade física para idosos. Demonstraram por meio de seus entendimentos a importância da educação permanente para uma prática mais abrangente, segura, eficaz, interdisciplinar e humanizada.

O Ministério da Saúde deixa claro que é essencial a questão das demandas para a formação e o desenvolvimento dos trabalhadores no SUS. Preconiza que estas não sejam definidas somente com base em uma lista de necessidades individuais de atualização e da capacidade de oferta e expertise de uma instituição de ensino, mas considerem, prioritariamente, os problemas cotidianos referentes à atenção à saúde e à organização do trabalho (Brasil, 2009).

Ao desenvolver ações de promoção da saúde voltadas para a população idosa, o processo educativo estratégico não deve ser restrito somente à graduação ou ao saber médico. Ele deve compreender níveis educacionais básicos e técnicos anteriores à graduação e estimular a educação permanente e continuada, com foco no trabalho interdisciplinar e em equipe (Motta e Aguiar, 2007). Este estudo apontou para essa direção, uma vez que 11 profissionais responsáveis por ações de promoção da saúde entrevistados $(39,3 \%)$ ocupavam cargos que não necessitam de formação superior.

Outro desafio apresentado à formação profissional em saúde relaciona-se à necessidade de adequação dos cursos técnicos, universitários, de pós-graduação e das residências para a formação de profissionais que possam atuar de forma democrática nas comunidades, inseridos na realidade local e no cotidiano dos serviços de saúde. Dessa forma, poderiam desenvolver propostas e ações condizentes com as reais necessidades de saúde da população por eles atendida.

Portanto, o desafio é desenvolver formas de descentralização e disseminação de processos pedagógicos, ou seja, processos que permitam aos trabalhadores, gestores e usuários ensinar e aprender em saúde. Porém, essa integração que deveria se concretizar no dia a dia do trabalho não acontece por diversas razões, sobretudo pela divisão fracionária do trabalho, pela normatização de tarefas preestabelecidas e pela fragmentação da assistência (Lima et al., 2010). Se os processos pedagógicos se transformarem em algo cotidiano na prática profissional de trabalhadores, gestores e serviços de saúde da APS, o SUS poderá se constituir em um verdadeiro sistema de saúde-escola.

\section{Considerações finais}

Pôde-se constatar, por meio deste estudo, a importância da atividade física para idosos como importante mecanismo de prevenção, promoção e educação 
em saúde. Porém, desenvolver ações de promoção de saúde para idosos é tarefa complexa, que exige dos profissionais habilidades e competências que vão além da atividade física propriamente dita. Identificar e classificar as necessidades de saúde da população idosa é importante para profissionais, estudantes e professores que atuam na ESF.

O que se observa atualmente nos serviços de saúde é que a maioria das ações são planejadas por alguns integrantes da equipe e pelos gestores, sem que haja a participação de todos os profissionais da rede e dos idosos. Não há envolvimento dos participantes e dos executores das ações no planejamento; assim, as reais necessidades de saúde podem não ser alcançadas.

O mesmo fenômeno pôde ser observado nas ações de promoção da saúde relacionadas às atividades físicas desenvolvidas pelos acadêmicos e docentes. Tratava-se de iniciativas pontuais, preconcebidas e com metodologias semiestruturadas que eram inseridas no cotidiano de uma UBS.

Transformar práticas de saúde baseadas na divisão de trabalho, na normatização de tarefas e, consequentemente, na fragmentação da assistência é tarefa complicada. A formação de recursos humanos deve pautar-se na compreensão do modelo biopsicossocial aplicado ao envelhecimento e na necessidade do trabalho interdisciplinar e multiprofissional, ou seja: deve-se colocar na agenda dos serviços de saúde práticas de assistência em que a intersetorialidade e a integralidade sejam marcas da atuação profissional das equipes. Desse modo, a educação permanente em saúde pode se apresentar como interessante ferramenta de capacitação para profissionais, estudantes e docentes responsáveis por ações de promoção da saúde.

\section{Colaboradores}

Paulo Henrique Vilela Oliveira de Sá concebeu e elaborou o projeto. Fez coleta, análise e interpretação dos dados, além da redação do manuscrito. Geraldo Cunha Cury orientou o trabalho, participando também da análise e interpretação dos dados. Fez a revisão crítica relevante do conteúdo intelectual. Liliane da Consolação Campos Ribeiro realizou a análise e interpretação dos dados, além da revisão crítica relevante do conteúdo intelectual. Os três autores participaram da aprovação final do artigo. 
Resumen El estudio tuvo como objetivo identificar y describir las acciones de promoción de la salud relacionadas con la actividad física de adultos mayores en las unidades básicas de salud, así como la percepción de los responsables sobre esta práctica. Se trató de una investigación transversal, de abordaje cuantitativo, realizado en Diamantina, estado de Minas Gerais, Brasil, en 2012. Se utilizó un instrumento con veintiocho afirmaciones, organizado en forma de escala Likert, durante las entrevistas con 28 responsables de tales acciones. Se cons-tataron 11 acciones educativas de promoción de la salud relacionadas con la actividad física para adultos mayores, cinco de responsabilidad de los profesores y alumnos de una institución pública de enseñanza y seis de responsabilidad de los profesionales de los equipos de salud de la familia. Las acciones observadas fueron "grupo de caminata y ejercicio (estiramientos)", "grupo de columna" y " Qigong". Los participantes demostraron comprensión sobre los beneficios de las actividades físicas para adultos mayores, el impacto de estas acciones en la salud pública y la necesidad de capacitación permanente. Palabras clave envejecimiento; educación en salud; educación continuada; actividad física; salud de la familia.

\section{Notas}

1 Universidade Federal dos Vales do Jequitinhonha e Mucuri, Pró-Reitoria de Extensão e Cultura, Diamantina, Minas Gerais, Brasil.

<pauloh.vilela@ufvjm.edu.br>

Correspondência: Rua Grupiara, 147-A, Bairro Vila dos Garimpeiros, CEP 39100-000, Diamantina, Minas Gerais, Brasil.

2 Universidade Federal de Minas Gerais, Faculdade de Medicina, Departamento de Medicina Preventiva e Social, Belo Horizonte, Minas Gerais, Brasil.

<geraldocunhacury@gmail.com>

3 Universidade Federal dos Vales do Jequitinhonha e Mucuri, Departamento de Enfermagem, Diamantina, Minas Gerais, Brasil.

$<$ liliane.consolacao@ufvjm.edu.br>

4 O presente artigo é parte da dissertação de mestrado profissional intitulada Ações educativas de promoção da saúde relacionadas à atividade física nas unidades básicas de saúde do município de Diamantina, Minas Gerais, de autoria de Paulo Henrique Vilela Oliveira de Sá, apresentada em 25 de julho de 2013 ao Programa de Pós-Graduação em Ensino em Saúde da Universidade Federal dos Vales do Jequitinhonha e Mucuri. 


\section{Referências}

BRASIL. Lei n. 10.741 , de $1^{\circ}$ outubro de 2003. Dispõe sobre o Estatuto do Idoso e dá outras providências. 2003. Disponível em: <www.planalto.gov.br/ccivil_03/Leis/2003/ L10.741.htm>. Acesso em: 7 jul. 2013.

Brasil. Ministério da Saúde. Secretaria de Gestão do Trabalho e da Educação na Saúde. Departamento de Gestão da Educação em Saúde. Política Nacional de Educação Permanente em Saúde. Brasília: Ministério da Saúde, 2009. (Série B. Textos Básicos de Saúde; Série Pactos pela Saúde 2006; v. 9). 64p. Disponível em: <http://bvsms.saude. gov.br/bvs/publicacoes/politica_nacional_ educacao_permanente_saude.pdf $>$. Acesso em: 7 jul. 2013.

CARDOSO, Ivana M.; MURAD, Ana L.G.; BOF, Sandra M. S. A institucionalização da educação permanente no Programa Saúde da Família: uma experiência municipal inovadora. Trabalho, Educação e Saúde, Rio de Janeiro, v. 3, n. 2, p. 429-439, 2005.

CONILL, Eleonor M. M. Ensaio histórico conceitual sobre a atenção primária à saúde: desafios para a organização de serviços básicos e da Estratégia Saúde da Família em centros urbanos no Brasil. Cadernos de Saúde Pública, Rio de Janeiro, v. 24, supl. 1, p. s7-s27, 2008.

GÓIS, Ana L. B; VERAS, Renato P. Informações sobre a morbidade hospitalar em idosos nas internações do Sistema Único de Saúde do Brasil. Ciência \& Saúde Coletiva, Rio de Janeiro, v. 15, n. 6, p. 2.859-2.869, 2010.

GONZAGA, Jozilma M. et al. Efeitos de diferentes tipos de exercícios nos parâmetros do andar de idosos. Revista Brasileira de Medicina do Esporte, São Paulo, v. 17, n. 3, p. 166-170, 2011.

INSTITUTO BRASILEIRO DE GEOGRAFIA E ESTATÍsTICA (IBGE). Pesquisa Nacional por Amostra de Domicílios (PNAD), 2014. 2014. Disponível em: <www.ibge.gov.br> Acesso em: 19 nov. 2014.
LIMA, Josiane V. C. et al. A educação permanente em saúde como estratégia pedagógica de transformação das práticas: possibilidades e limites. Trabalho, Educação e Saúde, Rio de Janeiro, v. 8, n. 2, p. 207-227, jul.-out. 2010.

MELO, Monica C. et al. A educação em saúde como agente promotor de saúde. Ciência \& Saúde Coletiva, Rio de Janeiro, v. 14, supl. 1, p. 1.579-1.586, 2009.

MOTTA, Luciana B.; AGUIAR, Adriana C. Novas competências profissionais em saúde e o envelhecimento populacional brasileiro: integralidade, interdisciplinaridade e intersetorialidade. Ciência \& Saúde Coletiva, Rio de Janeiro, v. 12, n. 2, p. 363-372, 2007.

PILGER, Calíope; MENON, Mario U.; MATHIAS, Thais A. F. Utilização de serviços de saúde por idosos vivendo na comunidade. Revista da Escola de Enfermagem da USP, São Paulo, v. 47, n. 1, p. 213-220, 2013.

RAMOS, Luiz R. Fatores do envelhecimento saudável em idosos residentes em centro urbano: Projeto Epidoso, São Paulo. Cadernos de Saúde Pública, Rio de Janeiro, v. 19, n. 3, p. 793-798, 2003.

ROCHA, Saulo V. et al. Fatores associados à atividade física insuficiente no lazer entre idosos. Revista Brasileira de Medicina do Esporte, São Paulo, v. 19, n. 3, p. 191-195, 2013.

ROECKER, Simone; MARCON, Sonia S. Educação em saúde na Estratégia Saúde da Família: o significado e a práxis dos enfermeiros. Escola Anna Nery Revista de Enfermagem, Rio de Janeiro, v. 15, n. 4, p. 701-709, 2011.

SÁ, Paulo H. V. O. Ações educativas de promoção da saúde relacionadas à atividade física nas unidades básicas de saúde do município de Diamantina, Minas Gerais. 2013. 49f. Dissertação (Mestrado Profissional em Ensino em Saúde) - Programa de Pós-Graduação em Ensino em Saúde, Universidade Federal dos 
Vales do Jequitinhonha e Mucuri, Diamantina, 2013. Disponível em: <http://acervo. ufvjm.edu.br:8080/jspui/handle/1/368>. Acesso em: 3 mar. 2016.

SALIN, Mauren S. et al. Atividade física para idosos: diretrizes para implantação de programas e ações. Revista Brasileira de Geriatria e Gerontologia, Rio de Janeiro, v. 14, n. 2, p. 197-208, 2011.

SANTOS, Patrícia L.; FORONI, Priscila M.; CHAVES, Maria C. F. Atividades físicas e de lazer e seu impacto sobre a cognição no envelhecimento. Medicina, Ribeirão Preto, v. 42, n. 1, p. 54-60, 2009.
SIQUEIRA, Fernando V. et al. Aconselhamento para a prática de atividade física como estratégia de educação à saúde. Cadernos de Saúde Pública, Rio de Janeiro, v. 25, n. 1, p. 203-213, 2009.

TESSER, Charles D. et al. Estratégia Saúde da Família e análise da realidade social: subsídios para políticas de promoção da saúde e educação permanente. Ciência \& Saúde Coletiva, Rio de Janeiro, v. 16, n. 11, p. 4.295-4.306, 2011.

VERAS, Renato P. Prevenção de doenças em idosos: os equívocos dos atuais modelos. Cadernos de Saúde Pública, Rio de Janeiro, v. 5, n. 10, p. 1.834-1.840, 2012.

Recebido em 17/10/2013

Aprovado em 19/11/2014 